\title{
Truckies and Health Promotion: Using the ANGELO Framework to Understand the Workplace's Role
}

\begin{tabular}{|r|l|}
\hline Journal: & International Journal of Workplace Health Management \\
\hline Manuscript ID & IJWHM-09-2017-0070.R1 \\
\hline Manuscript Type: & Research Paper \\
\hline Keywords: & $\begin{array}{l}\text { Health Promotion, Workplace Health, Workplace Wellness, Organizational } \\
\text { change, truck drivers, ANGELO framework }\end{array}$ \\
\hline \multicolumn{2}{|l}{} \\
\hline
\end{tabular}

SCHOLARONE ${ }^{m}$

Manuscripts 


\begin{abstract}
Purpose: Workplaces are challenging environments which place workers at risk of obesity. This is particularly true for Australian road transport industry workplaces. The Analysis Grid for Environments Linked to Obesity (ANGELO) Framework is a public health tool which can be used to conceptualise obesogenic environments. It suggests workplaces have a variety of roles (in the physical, economic, political and sociocultural domains) in responding to obesity in transport industry workplaces. This paper presents findings which explore this idea.
\end{abstract}

Methodology: The project used a mixed-methods approach located within a Participatory Action Research (PAR) framework, to engage workplace managers and truck drivers in the implementation and evaluation of workplace health promotion strategies. The project involved 6 transport industry workplaces in Queensland, Australia.

Findings: This study found transport industry workplaces perceive themselves to have an important role in addressing the physical, economic, political and sociocultural aspects of obesity, as per the ANGELO Framework. However, transport industry employees - specifically, truck drivers - do not perceive workplaces to have a major role in health; rather, they consider health to be an area of personal responsibility.

Practical implications: Balancing the competing perceptions of truck drivers and workplace managers about the workplace's role in health promotion is an important consideration for future health promotion activities in this hard-to-reach, at-risk population.

Originality / Value: The use of the ANGELO framework allows the conceptualisation of obesity in a novel workplace context.

Keywords: health promotion, workplace, workplace culture, workplace policy, organizational change, truck drivers, hard-to-reach, ANGELO framework, obesity

Article Classification: Research Paper 


\section{Introduction}

Rates of obesity - defined as a body mass index (BMI) of $30 \mathrm{~kg} / \mathrm{m}^{2}$ [World Health Organisation, 2015] - are at 'epidemic' levels in Australia. Almost two in three Australian adults, or $63 \%$ of the adult population, were overweight or obese in 2012 [Australian Institute for Health and Welfare, 2012]. Wong et al. [2014] report that obesity places $70 \%$ of workers in the Australian transport industry at increased risk of chronic disease. When compared with other Australian workers, those in the transport industry have the highest incidence of health risk due to waist circumference and the second highest incidence of overweight or obesity measured by BMI [Council on Federal Financial Relations, 2010].

It is well-established in the literature that workplaces, and particularly blue-collar workplaces, are obesogenic environments [Blackburn, 2009; Escoffery et al., 2011; Davis et al., 2014; Raine et al., 2014]. For truck drivers in particular, the limitations of their workplace negatively impact health behaviour. The sedentary nature of the job, in combination with long work hours, restricts truck drivers' capacity to engage in physical activity and limits access to healthy food options [Dahl et al., 2009; Lane et al., 2009; Olson et al., 2009]. The complex nature of transport industry workplaces, and the challenges this presents to effective workplace health promotion, is reflected in both Australian and international literature [Hill et al., 2015].

Obesity poses significant costs to employers, particularly in terms of increased rates of absenteeism, productivity loss and workers' compensation claims [Blackburn, 2008; Heinen \& Darling, 2009]. The scale and complexity of the obesity epidemic in developed nations demands a multifaceted response from all sectors of society, including workplaces [ Heinen \& Darling, 2009]. The presence of natural social networks, the potential to reach a large audience and the amount of time people, particularly truck drivers, spend at work mean workplaces are now widely recognised as effective platforms for health promotion [Apostolopoulos et al., 2012]. However, there is a paucity of evidence for the role of blue-collar workplaces, and specifically those in the Australian road transport industry, in workplace health promotion to combat obesity. This paper addresses this issue.

\section{Methods}

The Queensland Transport Industry Workplace Health Intervention was a settings-based Participatory Action Research (PAR) project. It involved the implementation of seven health promotion interventions for obesity in selected Australian transport industry workplaces. Ethical approval for this project was obtained from the Queensland University of Technology Human Research Ethics Committee (Approval Number QUT 1300000412).

The project worked with six workplaces from five different transport industry organisations in the south-east Queensland region over a two-year period. These organisations were identified because they were known to the research team, or because they were members of the Australian Trucking Association's TruckSafe program (which sets industry standards to promote a safe, professional trucking industry in Australia). At each workplace a manager - who had the 
ability to either make decisions or talk directly to a decision-maker - worked with the research team to facilitate the delivery of interventions, and to assist with access to truck drivers. The demographics of the participating workplaces, workplace managers and truck drivers are described in detail elsewhere [citation withheld until manuscript acceptance].

The project used a range of methods located within a Participatory Action Research (PAR) approach to engage workplace managers and truck drivers in the implementation and evaluation of workplace health promotion strategies. PAR is a recognised public health methodology [citation withheld until manuscript acceptance]. It is used because evidence suggests the involvement of stakeholders in health promotion projects through decision-making, planning and implementation is more likely to produce meaningful change [Judd \& Keleher, 2010]. .

Through the PAR process, at the beginning of the project a total of seven workplace health promotion interventions were developed: (1) healthy eating posters displayed in workplaces, (2) a healthy options vending machine for workplaces, (3) free fruit supplied to drivers at their workplaces' expense, (4) a ten thousand steps workplace challenge, (5) healthy eating and / or physical activity 'toolbox talk' presentations delivered by workplaces, (6) personalised health messages distributed by workplaces, and (7) the Truckin' Healthy Facebook webpage promoted by workplaces. Each workplace implemented at least three and up to four of these interventions during a six-month intervention period. Workplace managers decided which interventions to implement based on their workplace's capacity, constraints such as time and cost, the availability of drivers and drivers' preferences. Researchers supported the workplace managers to implement chosen interventions by providing resources (e.g. pre-prepared posters, Powerpoint slides, health messages, etc.) and practical guidance.

Using the PAR processes, data collection was undertaken at each workplace at three timepoints: pre-intervention, post-intervention (three months after intervention implementation commenced) and final follow-up (six months after intervention implementation commenced). Both quantitative and qualitative data were collected. This paper reports data collected at all three time points which identifies workplace managers' and drivers' perceptions about the role of transport industry workplaces in health promotion interventions. At each time point, workplace managers and truck drivers were invited to: (1) complete a short, mixed-methods survey, and (2) participate in an interview and / or focus group; informed consent was obtained.

The surveys were anonymous, paper-based multi-item tools, comprised of simple numerical scales, checkboxes and short-response questions. Workplace managers were responsible for distributing surveys, collecting these and returning them to the research team via email or post. The surveys were designed to obtain information about participants' health, healthy eating and physical activity experiences, perceptions about healthy eating and physical activity at work, and general thoughts about workplace health, and to detect changes in these over time. Across the study period, a total of 68 surveys were returned from workplace managers and truck drivers.

The interviews and focus groups were anonymous, confidential and audio-recorded. They were conducted at a time and location convenient to the participants - usually at their workplace -and 
they lasted between 20 and 70 minutes each. The questions asked were open-ended and semistructured. They were developed by the research team to encourage participants to share their experiences, attitudes and values, etc., about workplace health promotion. Across the study period, each of the 6 workplace managers and approximately 30 truck drivers participated in at least one and up to three interviews and / focus groups.

This paper focuses on reporting qualitative data collected in the surveys, interviews and focus groups. An inductive process of coding and theming was undertaken to analyse this data. Using a standard coding-and-theming approach, key quotes - from the surveys, and the audiorecordings of the focus groups and interviews - were transcribed, read several times and then organised by one member of the project team into a framework (described following). This coding-and-theming process was repeated until each of the quotes settled into a discrete section of the framework. The organisation of the quotes into the framework was critically discussed, and agreed upon, with other project team members.

During analysis, data was organised into the Analysis Grid for Environments Linked to Obesity (ANGELO) Framework. The ANGELO Framework is a tool used in public health research to conceptualise obesogenic environments [Swinburn et al., 1999]. The Framework suggests the factors contributing to the obesogenicity of certain environments can be classified into one of four different types:

- Physical - related to what is available.

- Economic - related to the costs.

- Political - related to the rules and regulations involved.

- Sociocultural - related to attitudes, values and beliefs.

[17].

The ANGELO Framework recognises workplaces as 'micro-environments', small and geographically-distinct environments which impact peoples' nutrition, physical activity and weight [Kirk et al., 2009]. At the micro-level, as in workplaces, the physical, economic, political and sociocultural factors contributing to obesity in these environments can be influenced [Vine et al., 2014]. The factors contributing to obesity in workplace environments which the research suggested can and should be influenced by workplaces have been organised into ANGELO framework in this paper.

\section{Findings}

This research suggests transport industry workplace managers and drivers perceive workplaces to have a variety of roles in responding to obesity through workplace health promotion interventions. The key themes to emerge from this research are presented in Figure 1:

$\rightarrow \quad$ INSERT FIGURE 1

The Physical Environment 
Access to healthy foods in the workplace. Drivers' access to healthy foods in the participating workplaces was a particular issue. Most of the workplaces had vending machines, canteens or tearooms which supplied drivers with cheap, unhealthy pre-packaged snack foods and sugary drinks. The workplace managers recognised drivers' access to foods in the workplace was a significant predictor of dietary intake at work. When discussing the variety of unhealthy foods to which drivers have access, one workplace manager observed, "They really don't have the choice" (TWP2140923).

Workplace managers recognised workplaces had a role in facilitating drivers' access to healthy foods and reducing their access to unhealthy foods in the workplace. Four of the six workplaces participated in an intervention to supply drivers with free fruit in the workplace, and drivers had a positive response to this intervention. One driver commented, "It became a bit of a staple for me" (TWP1141212). Workplace managers identified the main reason for drivers' high level of engagement in this intervention was that the fruit was provided at no cost. One workplace manager observed, "It was only the fact that it was being given to them free that they chose it" (TWP1142309). Workplace managers reflected on a range of strategies to facilitate drivers' engagement in this intervention, including placing fruit in convenient locations, offering variety and providing types of fruit easy to eat 'on the move'. Some workplaces took the 'free fruit' intervention further by removing unhealthy food and providing other healthy options in the workplace. One workplace manager explained, "We did stop supplying biscuits completely... and we do wraps instead of sandwiches... With our barbecues, too, we started offering up juices instead of soft-drinks... We even offer gluten-free options" (TWP6141217).

In the context of a mobile workforce without a fixed workplace or schedule, access to healthy foods can pose considerable difficulties. One workplace manager reported, "One of [the drivers] bought up the fact that he can't gain access to the kitchen facilities at our Sydney depot after hours... so he has to go out and buy something" (TWP6141217). Recognising the problems associated with unhealthy food options at truck stops, this workplace modified work processes to allow drivers after-hours access to kitchen facilities at this depot. Another workplace manager highlighted the difficulties of engaging highly mobile drivers in the 'free fruit' intervention stating, "There's one driver who came in today, I haven't seen that driver for seven weeks... How would I get free fruit to him?" (TWP4140925). Long, irregular work hours were identified as another constraint to drivers accessing healthy foods at work. When asked about what could be changed by workplaces to improve drivers' intake of healthy food at work one driver simply suggested, "Provision for proper meal breaks" (TWP635). Whilst rest breaks are mandatory for Australian truck drivers, spare time on the job is often spent in conditions restricted by responsibilities for vehicle and cargo, particularly if the truck is carrying livestock. Workplaces may have a role in addressing this through policy, a topic discussed in depth later in this paper.

Access to opportunities for physical activity in the workplace. Access to opportunities for physical activity in the participating workplaces was challenging. Workplace managers identified considerable, largely unalterable barriers to drivers' engagement in physical activity at work. This included the inherent sedentariness of the job and the widespread use of automated 
equipment in workplaces. One workplace manager explained, "At the moment they get out of the truck, they pull a chain, they get back in the truck, they drive off. They don't even need to get out of the truck at the wharf, everything's done from the controls in the cab. So there's really no requirement to get out and walk around" (TWP1142309). Occupational health and safety guidelines restricting drivers' movement around truck yards were another barrier to engagement in physical activity in the workplace. The only intervention in this project to address workplace physical activity - the ten thousand steps challenge - had very low levels of uptake among both workplaces and drivers.

Due to the substantial barriers associated with engaging in physical activity at work, workplace managers instead focused on supporting their drivers to be physically active outside of the workplace. For example, one workplace manager reported, "One thing we have seen an increase in is drivers asking operations for different hours, to work in with their exercise plans... We try to accommodate them when we can" (TWP6141217). This is particularly important given around one-quarter of participating drivers reported some, most or all of their daily physical activity was done outside of their workplace (specifically, travelling to and from work). However, drivers did identify that workplaces had a role in providing infrastructure to participate in physical activity in the workplace. When asked what could be changed by their workplaces to increase their physical activity at work, one driver responded, "Put a gym in, [or provide a] personal trainer" (TW538) - though the financial feasibility of such interventions is unclear if there is an expectation that the workplace would pay.

Drivers' access to workplaces and interventions. The problems associated with access to workplace health promotion interventions for a highly mobile workforce of drivers was referred to earlier. One workplace manager explained, "They're all over the place. You're lucky to see these guys once a week, once a month... We can never get them all together at the one time" (TWP3141212). Because workplace managers perceived themselves to have a role in workplace health promotion, they recognised a responsibility for facilitating their drivers' access to workplace health promotion interventions and employed a variety of strategies to do so. One workplace manager commented on the toolbox talks intervention that, "I could only grab drivers when I could get them" (TWP4140925) and another said, "You've nearly got to do it individually" (TWP5140919). A third explained another strategy, noting "They have to be here at the meeting and they have to sign the tool box sheets at the end of each tool box to say they were there" (TW1140923). Providing drivers' with access to workplace health promotion interventions was identified by workplace managers as important. One workplace manager explained, "[They say], 'Just tell me what you want me to do!'” (TWP1140923).

\section{The Economic Environment}

Workplaces subsidising cost of intervention implementation. Workplace managers consistently identified workplace subsidies to the cost of intervention implementation as essential to facilitating drivers' engagement in interventions. One workplace manager commented about the 'free fruit' intervention, "They wouldn't have purchased it themselves" (TWP1142309). Workplace managers recognised drivers simply could not afford, or would not prioritise, 
purchases of equipment necessary to engage in certain interventions. One workplace manager, reflecting about the ten thousand steps challenge, said "I don't think they were interested in paying for a step-counter themselves" (TW1140923). Another, commenting on the social media intervention, said, "You'd have to have a [smart] phone... They cost $\$ 700$, a lot of drivers wouldn't have $\$ 700$, you've got to remember that" (TWP4140925).

However, workplace managers were uncertain about the extent to which workplaces should, or indeed could, subsidise intervention implementation. One workplace manager commented about the 'free fruit' intervention, "They [management] told us to stop it... It [was] a couple of hundred dollars a week for half a dozen blokes... The cost was starting to go out [of hand]" (TWP2140923). Uncertainty about ongoing financial commitment to the interventions was one of the primary challenges to intervention sustainability in many workplaces. When asked about ongoing interventions one workplace manager explained, "Anything that has to be purchased... I'm not sure I'm going to get that authorised" (TW1140923).

\section{The Political Environment}

Workplace policies for health. None of the participating workplaces had pre-existing policies relating to drivers' health and most met only basic, mandatory occupational health and safety requirements. However, many of the workplace managers believed the only way to promote drivers' sustained engagement in workplace health promotion activities was via workplace policy. One workplace manager reflected, "Unless [the company] turns around and says, 'We're going to make this a company policy', I don't know..." (TWP2140923). Drivers also perceived policies to be important to health at work. When asked what could be changed by workplaces to increase drivers' physical activity at work, one driver wrote, "They could enforce mandatory rest breaks not to sleep, but walk around" (TWP635). Drivers' perceived importance of enforced meal breaks was highlighted earlier.

Transport industry workplaces are already bound by a variety of policies and underpinning legislation related to occupational health and safety. Workplace managers were concerned with the effect the introduction of new policies may have on drivers. One workplace manager noted, "They're pretty self-sufficient... If you say, 'Now everyone take an apple before you go on your trip', they'd say, 'Ah, don't tell me what to do'" (TWP5140909). Workplace managers reflected existing workplace policies negatively impacted on drivers' capacity to engage with some interventions. When asked about the reasons why his workplace did not implement the social media intervention, one workplace manager said, "That was when we had a 'no Facebook' policy" (TWP2140923).

\section{The Sociocultural Environment}

Workplace culture. As reported elsewhere, the improvements in drivers' health knowledge, health behaviour and self-reported health outcomes in this project were underpinned by significant positive changes in the culture of participating workplaces. Initially, a pervasive masculine culture in combination with the contextual constraints on drivers discussed earlier 
prevented many drivers from engaging in healthy behaviours. For example, when asked about consumption of fruit one driver commented, "A lot of men will not go to the supermarket specifically to buy fruit", and another explained, "When you're getting it [fruit] for yourself, you're not going to bother" (TWP1141212). However, when asked about the 'free fruit' intervention another driver commented, "The free fruit, that was encouraging when [the workplace manager] did that... I started bringing a bit more fruit myself" (TWP1141212). A number of the workplace managers themselves became actively involved in the interventions, thus effectively leading by example. One workplace manager reported, "I bring a fruit salad every day now; I've lost nearly 20 kilos" (TWP1141212). Peer support - or perhaps pressure - among drivers for healthy behaviour was an important influence in creating positive workplace cultures. One workplace manager commented, "The discussion in the drivers' room - their jokes, like 'Oh, mate, you'd better slow down your Coke; look at how much [sugar] is in that drink you just had" (TWP141217).

Positive shifts in workplace culture were demonstrated in other ways, including by the integration of new health interventions into existing workplace activities. One workplace manager gave the following example: "We were able to use a lot of the slideshows [developed by the research team]... in our existing tool box talks" (TWP6141217). The contextualisation of standardised interventions such as the tool box talks contributed to this positive shift in workplace culture. One workplace manager said, "We'd copy the posters themselves and put them onto the tool box as well. It makes it easier" (TWP6141217). Another factor contributing to a positive shift in workplace culture was the workplace managers' demonstrated commitment to sustaining - and even 'going the extra mile' in the delivery of - the interventions. One workplace manager commented, "[The] free fruit will still be going when we remember to fill the bowl up; the posters will be up forever" (TWP5140909). Another workplace manager said, "With the sugary drinks [poster], we put a display there to make it more visual... a glass jar [showing] how much sugar was in the can" (TWP141217).

Multisectorial support for workplaces. Workplace managers identified the support of the research team as pivotal to their ability to implement workplace interventions. One workplace manager commented, " $[W e]$ probably wouldn't have thought of going down the poster path if you hadn't provided them" (TWP2140923). About the tool box talks another said, "They would be something you wouldn't be able to do [without external support]" (TWP5140919). Workplace managers perceived the support of the government to be important. One workplace manager commented, "It's only a matter of the government getting behind you" (GBFF140919).

\section{Drivers' Perceptions of Responsibility}

Interestingly, drivers do not perceive workplaces to have a major role in or responsibility for health. The post-intervention survey asked drivers what could be changed by workplaces to (a) improve the intake of healthy food and (b) increase physical activity at work. One driver reflected "All down to me! I need to make the healthy choice instead of fast food" (TWP119). Another said "Doing it for yourself" (TWP58) and a third commented "Personal choice" (TWP25). Another driver reflected "I believe it is up to me to change" (TWP220). While 
workplace managers did perceive workplaces to have a role in drivers' health, one workplace manager commented, "It's only going to work if [the drivers] want to change" (TWP5140919).

\section{Discussion}

Workplaces recognise they have a role, to some degree, in creating change to modify obesogenic factors in the workplace environment. Specifically, workplaces identify a role in promoting drivers' access to healthy foods, reducing or removing unhealthy food options and providing opportunities for engagement in physical activity. The importance of a healthy workplace environment to employees' health in obesity prevention in blue-collar workplaces is well-established in the literature [Escoffery et a., 2011; DeJoy et al., 2012]. The novel finding from this study is that some workplaces perceive they have a role in implementing processes in the workplace which may facilitate drivers' engagement in physical activity outside of work. Chief among these processes was accommodating changes to drivers' work rosters to support engagement in physical activity. However, it is important to acknowledge this occurred in just one of the larger workplaces. Such an intervention is likely to be less feasible in smaller workplaces with reduced capacity for flexibility in work schedules.

Workplaces recognised that subsidising workplace health promotion interventions was important in facilitating drivers' participation. However, workplaces were uncertain about the extent to which they should, or indeed could, subsidise certain intervention activities and the sustainability of these subsidies. Other research on workplace health promotion interventions suggests workplaces' financial commitment to health promotion interventions is considerably dependent on individual workplace context [Safe Work Australia, 2008]. This was evident in this project, with the extent of each workplace's financial commitment to the project determined by factors such as organisation size, participant numbers and the types of interventions implemented. Even in two of the larger workplaces involved in the project (which made the most significant and sustained financial commitment to interventions), the costs involved in a number of the interventions impaired and even prohibited implementation and sustainability. This was particularly true of the free fruit intervention (which many workplaces implemented but were unable to sustain), the ten thousand steps challenge (which necessitated the purchase of pedometers) and the 'healthy options' vending machine (which required considerable financial outlay). These interventions could be important to fostering a healthful workplace environment, but few workplaces trialled them due to financial constraints.

As suggested by Blackburn [2008], to promote workplaces' commitment to funding health promotion interventions there is a clear need for more research evidence on the cost effectiveness of different interventions. Cost effectiveness evaluations were not undertaken in this project, because the diversity of the workplaces involved and the small number of participating drivers would make such evaluations highly complex. However, these are an important consideration for similar future research. In terms of 'costs' associated with workplace health promotion interventions, responses from workplace managers indicate they perceive the concept of 'cost' quite narrowly, as simply the financial outlay involved in implementing an intervention. To promote workplaces' financial commitment to health promotion interventions, it 
is also necessary to reframe the question of what constitutes 'cost effectiveness', with workplaces factoring in indirect savings related to reduced absenteeism, productivity loss and workers' compensation claims, etc. [Blackburn, 2008].

While none of the workplaces had pre-existing policies relating to drivers' health, workplaces identified they had an important role in developing such policies to promote drivers' engagement in workplace health promotion activities. This finding is supported by other research into health promotion interventions in blue-collar workplaces [DeJoy et al., 2012]. Policy, including workplace policy, and particularly policies which address the underlying causes of obesity, are now recognised as a key strategy in obesity reduction and prevention in workplaces [Raine et al., 2014]. Although Australian workplaces are strongly encouraged to adopt such policies, these are not mandatory [Safe Work Australia, 2008]; indeed, the only mandatory health policies for Australian trucking workplaces, reflected in relevant legislation, are those related to driver fatigue management [National Heavy Vehicle Regulator, 2017]. Interestingly, the workplace managers participating in this study were concerned that obesity management policies may be perceived as paternalistic, and in fact have a negative impact on drivers' engagement with workplace health promotion interventions. This idea was not widely explored in this study, but may be an important topic for future research.

The finding that drivers do not perceive workplaces to have a major role in health, and this is instead solely their own personal responsibility, brings an added level of complexity to this discussion. This finding is supported by other research, which suggests the majority of peoplemore than 70\% in one North American study [Raine et al., 2014] - view both the causes and management of obesity as being issues of personal responsibility [Ices \& Sharma, 2009; Greener et al., 2010]. As noted by McKay [2008], paternalistic approaches to obesity - including workplace and industry policies which diminish or remove personal responsibility - are particularly reviled in the Australian context. Research suggests policies related to obesity reduction and prevention which receive the strongest endorsement are those focused on individual responsibility for behaviours, including environmental change policies which facilitate healthy obesity-related behaviours [ Niederdeppe et al., 2008; Raine et al., 2014]. In Australia, legislation which frames obesity as the responsibility of individuals and which is aimed at facilitating healthy obesity-related behaviours - including legislation related to the disclosure of nutritional information on packaging and advertising regulations - is generally supported by the public [McKay, 2008]. This project did not involve the implementation of policies related to workplace health promotion interventions, however this may be a topic for future research.

The workplaces in this project recognise they have an important role in fostering a workplace environment conducive to healthy behaviour, not only by enabling access to healthy food and opportunities for physical activity but also in less-tangible ways - including through the fostering of a healthy work culture. The importance of a healthy work culture in obesity prevention in bluecollar workplaces is well-established in the literature [Heinen \& Darling, 2009; Judd \& Keleher, 2010; Escoffery et al., 2011; DeJoy et al., 2012]. In this project, a healthy workplace culture was fostered in three key ways - by managers demonstrating commitment to drivers' health, by managers leading by example and through driver/driver peer support. The importance of peer 
support in particular has been demonstrated by other studies on health interventions in blue collar workplaces [Escoffery et al., 2011].

In addition, workplaces recognise the importance of multisectorial support and identified their role in engaging such support. Again, the importance of a collaborative approach to obesity prevention in blue-collar workplaces is well-established in the literature [Blackburn, 2009; Escoffery et al., 2011; Davis et al., 2014; Raine et al., 2014]. Workplaces identified the support of the research team as particularly important. Equivalent support for workplaces may involve governmental, non-governmental, industry and wider community organisations [Raine et al., 2014].

\section{Conclusion}

The use of the ANGELO framework allowed the conceptualisation of obesity in a novel workplace context. This paper suggests that transport industry workplaces do perceive themselves to have an important role in addressing the physical, economic, political and sociocultural aspects of obesity. However, drivers do not perceive workplaces to have a major role in health because this is an area of personal responsibility. Balancing these competing perceptions is an important consideration for future health promotion activities in this hard-toreach, at-risk population. 


\section{References}

Apostolopoulos, Y, Sonmez, S, Shattell, M \& Belzer, M.H. (2012). Environmental determinants of obesity-associated morbidity risks for truckers. International Journal of Workplace Health and Safety, 5(1), 120-138. doi: 10.1108/17538351211239162

Australian Institute of Health and Welfare. (2012). Overweight and obesity. Australian Institute of Health and Welfare. Available: http://www.aihw.gov.au/overweight-and-obesity/

Blackburn, D. (2008). Making obesity everybody's business: What is the employer's role? Obesity Management, 4(4), 169-175. doi: 10.1089/obe.2008.0205.

Council on Federal Financial Relations. (2010). Implementation plan for the healthy workers initiative. Council on Federal Financial Relations. Canberra, Australia

Crane, P \& O’Regan, M. (2010). On PAR: Using Participatory Action Research to Improve Early Intervention. Commonwealth of Australia, Canberra, ACT.

Dahl, S, Kaerlev, L, Jensen, A, Tuchsen, F, Hannerz, H, Neilsen, PS \& Olsen, J. (2009). Hospitalization for lifestyle related diseases in long haul drivers compared with other truck drivers and the working population at large. Work, 33(1), 345-353.

Davis, J, Clark, B, Lewis, G \& Duncan, I. (2014). The impact of a worksite weight management program on obesity: A retrospective analysis. Population Health Management17(5), 265271.

DeJoy, DM, Wilson, MG, Padilla, HM, Goetzel, RZ, Parker, KB, Della, LJ \& Roemer, EC. (2012). Process evaluation results from an environmentally focused worksite weight management stud. Health Education and Behaviour, 39(4), 405-418. doi: 10.1177/1090198111418109

Escoffery, C, Kegler, MC, Alcantara, I, Wilson, M \& Glanz, K. (2011). A qualitative examination of the role of small, rural worksites in obesity prevention. Preventing Chronic Disease 8(4), 7587.

Greener, J, Douglas, F \& van Teijlingen, E. (2010). More of the same? Conflicting perspectives of obesity causation and intervention amongst overweight people, health professionals and policy makers. Social Science and Medicine, 70(7), 1042-1049.

Heinen, L \& Darling, H. (2009). Addressing obesity in the workplace: The role of employers. The Milbank Quarterly, 87(1), 101-122. doi: 10.1111/j.1468-0009.2009.00549.x

Hill, MA, Sendall, MC \& McCosker, LK. (2015). Truckies and health promotion: A 'hard-to-reach' group without a 'proper' workplace. Journal of Health, Safety and Environment, 31(2), 93-105. 
Ickes, M \& Sharma, M. (2009). Worksite health promotion: A practical strategy for obesity prevention. American Journal of Health Studies, 24(3).

Judd, J \& Keleher, H. (2010). Reorienting health services in the Northern Territory of Australia: A conceptual model for building health promotion capacity in the workforce. Global Health Promotion, 20(2), 53-63.

Kirk, SFL, Penney, TL \& McHugh, TLF. (2009). Characterising the obesogenic environment: The state of the evidence with directions for future research. Obesity Reviews, 11, 109-117.

Layne, DM, Rogers, B \& Randolph, SA. (2009). Health and gender comparisons in the long haul trucking industry: A pilot study. American Association of Occupational Health Nurses Journal, 57(1), 405-413. doi: 10.3928/08910162-20090916-01

McKay, S. (2008). Legislative solutions to unhealthy eating and obesity in Australia. Public Health, 125, 896-904.

National Heavy Vehicle Regulator. (2017). Heavy Vehicle National Law and Regulations. National Heavy Vehicle Regulator. Available: https://www.nhvr.gov.au/law-policies/heavyvehicle-national-law-and-regulations

Niederdeppe, J, Robert, SA \& Kindig, DA. (2008). Qualitative research about attributions, narratives and support for obesity policy. Prevention of Chronic Disease, 8(2), A39.

Olson, R, Anger, WK, Elliot, DL, Wipfli, B \& Gray, M. (2009). A new health promotion model for lone workers: Results of the safety and health involvement for truckers (SHIFT) pilot study. Journal of Occupational and Environmental Medicine, 51(1), 1233-1246. doi: 10.1097/JOM.0b013e3181c1dc7a

Raine, KD, Nykiforuk, ClJ, Vu-Nguyen, K, Nieuwendyk, LM, VanSpronsen, E, Reed, S \& Wild, TC. (2014). Understanding key influencers' attitudes and beliefs about healthy public policy change for obesity prevention. Obesity, 22(11), 2426-2433. doi: 10.1002/oby.20860

Safe Work Australia. (2008). Overweight and obesity: Implications for workplace health and safety and workers compensation. Available:

http://www.safeworkaustralia.gov.au/sites/SWA/about/Publications/Documents/326/Overweight AndObesity_Implications_WorkplaceHealthSafety_WorkersCompensation_2008_PDF.pdf

Swinburn, B, Egger, G \& Raza, F. (1999). Dissecting obesogenic environments: The development and application of a framework for identifying and prioritizing environmental interventions for obesity. Preventive Medicine, 29(1), 563-570. 
Vine, MM \& Elliott, SJ. (2014). Exploring the school nutrition policy environment in Canada using the ANGELO Framework. Health Promotion Practice, 15(3), 331-339. doi:

$10.1177 / 1524839913498087$

Wong, JYL, Gilson, ND, Bush, RA \& Brown, WJ. (2014). Patterns and perceptions of physical activity and sedentary time in male transport drivers working in regional Australia. Australian and New Zealand Journal of Public Health. 38(1), 314-320. doi: 10.1111/1753-6405.12214

World Health Organisation. (2015). Overweight and obesity. World Health Organisation. Available: http://www.who.int/mediacentre/factsheets/fs311/en 
PHYSICAL

ECONOMIC

POLITICAL

SOCIOCULTURAL

- Access to

- Workplaces

healthy (and unhealthy)

foods in the

workplace

- Access to opportunities for physical activity in the workplace

- Drivers' access to workplaces

and interventions subsidising cost of intervention implementation

- Workplace policies for health
- Workplace culture

- Multi-sectorial support for workplaces

FIGURE 1. Use of the ANGELO Framework to conceptualise the role of road transport industry workplaces in responding to obesity 